
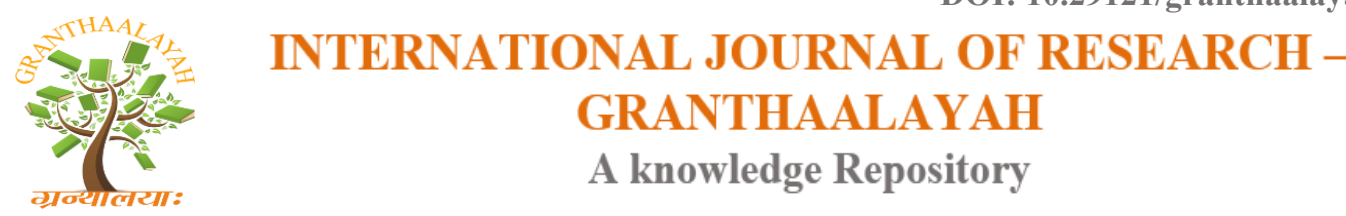

Science

\title{
PHYSICO-CHEMICAL EVALUATION OF TOMATO HYBRID DERIVATIVES FOR PROCESSING SUITABILITY
}

\author{
Fikreyohannes Gedamu Mihretu ${ }^{* 1}$, M.N. Bhalekar ${ }^{2}$ \\ ${ }^{* 1}$ School of Plant Sciences, Haramaya University, P.O.B. 56, ETHIOPIA \\ ${ }^{2}$ Mahatma Phule Agricultural University, Rahuri, 413722 Dist. Ahmednagar, Maharashtra,
} INDIA

\begin{abstract}
Investigation was carried out to evaluate F5 and F6 generations of tomato hybrid derivatives for its physicochemical characters and its suitability for processing during spring-summer 2009/10 and 2010/11 at Vegetable Research area, Mahatma Phule Agricultural University, Rahuri. F5 and F6 generations of two crosses (M-3-1 $x$ H-24 and 87-2 $x$ 18-1-1) and two standard checks ('Bhagyashree' and 'Dhanashree') were evaluated for processing suitability in randomized block design in three replications. There were significant differences in all the fruit physical characters studied. Significantly highest polar and equatorial diameters, shape index of fruit, pericarp thickness were recorded in both F5 and F6 generation. Biochemical composition and processing qualities of tomato genotypes in both F5 and F6 generations showed significant differences except fruit juice per cent in F6 generation. Major processing quality characters maximum recorded in F5 and F6 generations were: total soluble solids, 5.03 (T18) and 5.17oBrix (T27); titratable acidity 0.6 per cent (T13) and 0.68 per cent (T24); low pH, 4.07 (T21) and 4.07 (T6); maximum ascorbic acid, 36.27 (T26) and 32.93 (T32) mg/100 g; fruit total sugars, 3.72 per cent (T6) and 3.71 per cent (T26); maximum lycopene in T26, which was 5.95 and $4.23 \mathrm{mg} / 100 \mathrm{~g}$; $\beta$-carotene, was highest in T32, 2.97 and 2.94 $\mathrm{mg} / 100 \mathrm{~g}$ in $\mathrm{F5}$ and F6 progenies.
\end{abstract}

Keywords:

biochemical composition, cross, fruits, generation(s), progenies.

Cite This Article: Fikreyohannes Gedamu Mihretu, and M.N. Bhalekar, "PHYSICOCHEMICAL EVALUATION OF TOMATO HYBRID DERIVATIVES FOR PROCESSING SUITABILITY" International Journal of Research - Granthaalayah, Vol. 4, No. 3 (2016): 131145.

\section{INTRODUCTION}

Tomato is a popular and highly consumed vegetable worldwide (Swamy and Sadashiva, 2007; Sharma et al., 2008). It is commonly used for table consumption as fresh or cooked dishes and for processing into several products such as paste, puree, ketchup, sauce or juice. The area under 
tomato crop is increasing and yields per unit area also increasing to considerable extent. The high produce of tomato often causes glut in the market. Since the tomato fruits are highly perishable, growers are obligated to sell their produce immediately after harvest resulting in lower incomes. Therefore, it is necessary to overcome the problem of excessive production during glut period. It can be facilitated very effectively with the processing of the tomato fruits, so as to stabilize the market prices in the interest of growers and to maintain a steady supply of tomatoes to the consumer in processed form.

Production of tomato fruit for processing is a specialized industry which requires desired qualities which are met only by certain varieties of tomato. The desirable qualities for a tomato fruit to be used for processing includes minimum number, 2-3 locules, pericarp thickness should be more than $0.5 \mathrm{~cm}$, oblong types with more polar diameter as they produce more pulp, uniformly red colour with more than $50 \mathrm{~g}$ fruit weight, small core, firm flesh, high total soluble solids (4- $8^{\circ}$ Brix), acidity not less than 0.4 per cent and more amount of ascorbic acid, lycopene and $\beta$-carotene content (Adsule et al., 1980; Tiwari et al., 2002). Quality of the processed products depend on the chemical compositions such as total soluble solids, sugars, acidity, ascorbic acid, lycopene and $\beta$-carotene which plays an important role in deciding the suitability of the genotypes for processing purpose (Balasubramanian, 1984; Swamy and Sadashiva, 2007).

There is limited iinformation on suitability of tomato genotypes for processing purpose. Hence this study was initiated with the objective to identify physiochemical properties of progenies in $\mathrm{F}_{5}$ and $\mathrm{F}_{6}$ generations for its suitability for processing.

\section{MATERIALS AND METHODS}

The study was conducted at All India Coordinated Research Project on Vegetable Crops, Department of Horticulture, Mahatma Phule Agricultural University, Rahuri, during springsummer season of 2009/10 and 2010/11.

\subsection{EXPERIMENTAL MATERIAL}

The seeds of tomato genotypes of $\mathrm{F}_{4}$ generation of crosses M-3-1 x H-24 (19 progenies) and 87-2 x 18-1-1 (11 progenies) were obtained from All India Coordinated Research Project on Vegetable Crops, Mahatma Phule Agricultural University, Rahuri. Standard checks 'Bhagyashree' and 'Dhanashree' were obtained from Tomato Improvement Scheme, Mahatma Phule Agricultural University Rahuri. Name of chemicals used for the study were: Sodium hydroxide $(\mathrm{NaOH})$, Phenolphthalein indicator, Fehling's solution A(copper sulphate, $\mathrm{CUSO}_{4} .5 \mathrm{H}_{2} \mathrm{O}$ ), Fehling's solution $\mathrm{B}$ (potassium sodium tartarate, $\mathrm{KNaC}_{4} \mathrm{H}_{4} \mathrm{O}_{6} .4 \mathrm{H}_{2} \mathrm{O}$ ), methylene blue indicator, hydrochloric acid $(\mathrm{HCl})$, lead acetate, potassium oxalate $\left(\mathrm{K}_{2} \mathrm{C}_{2} \mathrm{O}_{4} \cdot \mathrm{H}_{2} \mathrm{O}\right)$, acetone, petroleum ether, anhydrous sodium sulphate $\left(\mathrm{NaSO}_{4}\right)$, magnesium oxide $(\mathrm{MgO})$, hexane, magnesium carbonate, diatomaceous earth (supercel), activated magnesia, metaphosphoric acid, l-ascorbic acid, dichlorophenol-indophenol, sodium bicarbonate. 


\subsection{EXPERIMENTAL DESIGN}

All the genotypes of $\mathrm{F}_{5}$ and $\mathrm{F}_{6}$ generations were laid out and evaluated in randomized block design with three replications and data were analyzed following procedures of Gomez and Gomez (1984) using MSTAT-C software.

\subsection{METHODS}

The plot size was $11.88 \mathrm{~m}^{2}(3.6 \mathrm{~m} \times 3.3 \mathrm{~m})$ and $4.86 \mathrm{~m}^{2}(1.8 \mathrm{~m} \times 2.7 \mathrm{~m})$ gross and net plot size, respectively. The plot size comprising four rows $0.9 \mathrm{~m}$ apart and $0.3 \mathrm{~m}$ part in the row with 44 plants in each plot. Ridges were opened at $90 \mathrm{~cm}$ apart. Plots were laid out and seedlings were transplanted in to the main field at $30 \mathrm{~cm}$ distance on one side of ridges on 08, December, 2009, one month after seedling emergence.Fertilizer was applied at the rate of 200, 100 and $100 \mathrm{~kg}$ NPK/ha of Urea (as source of N), Phosphorus (P) and Potassium (K), respectively. Full dose of farm yard manure, $\mathrm{P}, \mathrm{K}$ and half dose of $\mathrm{N}$ were applied before transplanting and remaining half dose of $\mathrm{N}$ were applied in three equal split doses at 20,40, and 60 days after transplanting as a top dressing. Other cultural practices such as irrigation, weeding, staking, tying, and earthing up were carried out as per the requirement of the crop. But no any pesticides applied to control diseases and insect pests in both the years. In 2010/11 cropping season, tomato seeds of $\mathrm{F}_{5}$ generations were sown on 01 , November 2010 on nursery beds and 3 weeks old seedlings (on $1^{\text {st }}$ December, 2010) were transplanted to the field. The plot size, spacing and method of planting, fertilizers application and other operations followed the previous year practices.

\subsection{DATA COLLECTION}

Samples were collected from research plots data were noted on the following physical fruit characters and biochemical compositions: fruit polar and equatorial diameter, fruit shape index, pericarp thickness, number of locules per fruit, fruit juice, total soluble solids, titratable acidity, $\mathrm{pH}$, ascorbic acid, sugars, lycopene and $\beta$-carotene. All the chemical analysis was done by following the procedures as suggested by Ranganna (1986) and A.O.A.C. (1990).

\section{RESULTS AND DISCUSSIONS}

\subsection{FRUIT POLAR AND EQUATORIAL DIAMETER}

The fruit polar diameter was significantly differed among progenies in both $\mathrm{F}_{5}$ and $\mathrm{F}_{6}$ generations (Table 1). In $\mathrm{F}_{5}$ generation, significantly maximum fruit polar diameter was $5.25 \mathrm{~cm}$ in $\mathrm{T}_{5}$, cross of M-3-1 x H-24 which was at par with $\mathrm{T}_{26}(5.23 \mathrm{~cm})$ and $\mathrm{T}_{28}(5.22 \mathrm{~cm})$ in cross 87-2 $\mathrm{x} 18-1-1$, and the minimum polar diameter was observed in $\mathrm{T}_{9}(3.45 \mathrm{~cm})$ which was at par with $\mathrm{T}_{22}(3.9 \mathrm{~cm})$. In $\mathrm{F}_{6}$ generation, significantly maximum polar diameter was $5.79 \mathrm{~cm}\left(\mathrm{~T}_{28}\right.$, cross of $87-2 \times 18-1-1)$ which was at par with $\mathrm{T}_{5}(5.6 \mathrm{~cm})$, and the minimum in $\mathrm{T}_{23}(4.00 \mathrm{~cm})$ which was at par with $\mathrm{T}_{9}(4.04 \mathrm{~cm})$. In both $\mathrm{F}_{5}$ and $\mathrm{F}_{6}$ generations, the mean polar diameter was found to be 4.55 and $4.81 \mathrm{~cm}$, respectively.

In fruit equatorial diameter there was significant differences among the genotypes in both generations (Table 1). The equatorial diameter was ranged from $5.69\left(\mathrm{~T}_{29}\right)$ and $5.84 \mathrm{~cm}\left(\mathrm{~T}_{28}\right)$ 
both in cross of 87-2 x 18-1-1) to 3.11( $\left.\mathrm{T}_{9}\right)$ and $3.29 \mathrm{~cm}\left(\mathrm{~T}_{9}\right)$ in cross of M-3-1 x H-24 in $\mathrm{F}_{5}$ and $\mathrm{F}_{6}$ generations, respectively. The mean fruit equatorial diameter was $4.49 \mathrm{~cm}\left(\mathrm{~F}_{5}\right)$ and $4.74 \mathrm{~cm}$ $\left(\mathrm{F}_{6}\right)$ generations.

\subsection{FRUIT SHAPE INDEX}

Tomato Fruit shape is one of the components of fruit firmness, which represents its ability for storability and shelf life. There were significant differences observed among the genotypes. Fruit shape index values ranged from $0.87\left(\mathrm{~T}_{29}\right)$ to $1.27\left(\mathrm{~T}_{11}\right)$ in $\mathrm{F}_{5}$ and from $0.91\left(\mathrm{~T}_{29}\right)$ to $1.27\left(\mathrm{~T}_{5}\right)$ in $\mathrm{F}_{6}$ generations. Accordingly, fruits were categorized in their shape index as suggested by Roy and Choudhary (1972). The majority progenies of crosses M-3-1 x H-24 and 87-2 x 18-1-1 in $\mathrm{F}_{5}$ and $\mathrm{F}_{6}$ generations had round shape (Table 1). The mean average value of fruit shape index was 1.02 in both $F_{5}$ and $F_{6}$ generations, which falls under round index. In this investigation $T_{5}, T_{9}$, $\mathrm{T}_{11}, \mathrm{~T}_{12}, \mathrm{~T}_{13}$ and $\mathrm{T}_{14}$ (cross of M-3-1 x H-24) and $\mathrm{T}_{22}$ and $\mathrm{T}_{24}$ (cross of 87-2 x 18-1-1) had oval shapes.

\subsection{FRUIT PERICARP THICKNESS}

Fruit keeping quality and shelf life depends on pericarp thickness and number of locules per fruit. The pericarp thickness in both $\mathrm{F}_{5}$ and $\mathrm{F}_{6}$ generations significantly varied and it was ranged from $3.8 \mathrm{~mm}\left(\mathrm{~T}_{22}\right.$ and $\mathrm{T}_{27}$, cross of 87-2 x 18-1-1) to $5.87 \mathrm{~mm}\left(\mathrm{~T}_{6}\right.$, cross of M-3-1 x H-24) in $\mathrm{F}_{5}$ progenies (Table 2). Similarly, in $\mathrm{F}_{6}$ generation progenies, pericarp thickness was significantly minimum $(4.03 \mathrm{~mm})$ in $_{15}$ and $\mathrm{T}_{23}$ at par with $\mathrm{T}_{24}(4.13 \mathrm{~mm})$, and significantly maximum (6.03 $\mathrm{mm})$ in $\mathrm{T}_{6}$ which was at par with $\mathrm{T}_{20}$ and $\mathrm{T}_{29}(5.7 \mathrm{~mm})$. Average pericarp thickness of tomato fruits were 4.86 and $4.92 \mathrm{~mm}$ in $\mathrm{F}_{5}$ and $\mathrm{F}_{6}$ generation progenies, respectively.

Table 1: Physical characters of tomato fruit

\begin{tabular}{|c|c|c|c|c|c|c|}
\hline \multirow[t]{2}{*}{ Treatment(T) } & \multicolumn{2}{|c|}{$\begin{array}{l}\text { Fruit polar } \\
\text { diameter }(\mathrm{cm})\end{array}$} & \multicolumn{2}{|c|}{$\begin{array}{l}\text { Fruit equatorial } \\
\text { Diameter }(\mathrm{cm})\end{array}$} & \multicolumn{2}{|c|}{$\begin{array}{l}\text { Fruit shape } \\
\text { index }\end{array}$} \\
\hline & $\mathrm{F}_{5}$ & $\mathrm{~F}_{6}$ & $\mathrm{~F}_{5}$ & $\mathrm{~F}_{6}$ & $\mathrm{~F}_{5}$ & $\mathrm{~F}_{6}$ \\
\hline $\mathrm{T}_{1}(\mathrm{M}-3-1 \times \mathrm{H}-24)$ & 4.52 & 5.18 & 4.90 & 5.56 & 0.93 & 0.93 \\
\hline $\mathrm{T}_{2}(\mathrm{M}-3-1 \times \mathrm{H}-24)$ & 4.71 & 4.80 & 4.81 & 5.23 & 0.98 & 0.92 \\
\hline $\mathrm{T}_{3}(\mathrm{M}-3-1 \times \mathrm{H}-24)$ & 4.69 & 4.47 & 4.91 & 4.74 & 0.95 & 0.94 \\
\hline $\mathrm{T}_{4}(\mathrm{M}-3-1 \times \mathrm{H}-24)$ & 4.53 & 4.87 & 4.69 & 4.98 & 0.97 & 0.98 \\
\hline $\mathrm{T}_{5}(\mathrm{M}-3-1 \times \mathrm{H}-24)$ & 5.25 & 5.60 & 4.25 & 4.39 & 1.25 & 1.27 \\
\hline $\mathrm{T}_{6}(\mathrm{M}-3-1 \times \mathrm{H}-24)$ & 4.39 & 5.49 & 4.48 & 5.35 & 0.99 & 1.03 \\
\hline $\mathrm{T}_{7}(\mathrm{M}-3-1 \times \mathrm{H}-24)$ & 4.28 & 4.68 & 4.34 & 4.87 & 0.99 & 0.96 \\
\hline $\mathrm{T}_{8}(\mathrm{M}-3-1 \times \mathrm{H}-24)$ & 4.38 & 4.51 & 4.82 & 4.80 & 0.91 & 0.94 \\
\hline $\mathrm{T}_{9}(\mathrm{M}-3-1 \times \mathrm{H}-24)$ & 3.45 & 4.04 & 3.11 & 3.29 & 1.11 & 1.23 \\
\hline $\mathrm{T}_{10}(\mathrm{M}-3-1 \times \mathrm{H}-24)$ & 4.52 & 4.55 & 4.47 & 4.44 & 1.02 & 1.02 \\
\hline $\mathrm{T}_{11}(\mathrm{M}-3-1 \times \mathrm{H}-24)$ & 4.64 & 4.78 & 3.66 & 4.38 & 1.27 & 1.11 \\
\hline $\mathrm{T}_{12}(\mathrm{M}-3-1 \times \mathrm{H}-24)$ & 4.27 & 4.80 & 3.70 & 4.02 & 1.15 & 1.19 \\
\hline $\mathrm{T}_{13}(\mathrm{M}-3-1 \times \mathrm{H}-24)$ & 4.72 & 5.03 & 3.88 & 4.46 & 1.22 & 1.13 \\
\hline $\mathrm{T}_{14}(\mathrm{M}-3-1 \times \mathrm{H}-24)$ & 4.94 & 4.77 & 4.51 & 4.49 & 1.10 & 1.07 \\
\hline $\mathrm{T}_{15}(\mathrm{M}-3-1 \times \mathrm{H}-24)$ & 3.99 & 4.23 & 3.90 & 4.45 & 1.03 & 0.95 \\
\hline
\end{tabular}




\begin{tabular}{lllllll}
$\mathrm{T}_{16}(\mathrm{M}-3-1 \times \mathrm{H}-24)$ & 4.64 & 5.47 & 4.76 & 5.78 & 0.98 & 0.96 \\
$\mathrm{~T}_{17}(\mathrm{M}-3-1 \times \mathrm{H}-24)$ & 4.56 & 5.35 & 4.19 & 5.25 & 1.08 & 1.02 \\
$\mathrm{~T}_{18}(\mathrm{M}-3-1 \times \mathrm{H}-24)$ & 4.03 & 4.25 & 4.23 & 4.43 & 0.96 & 0.96 \\
$\mathrm{~T}_{19}(\mathrm{M}-3-1 \times \mathrm{H}-24)$ & 4.82 & 4.88 & 4.84 & 5.01 & 1.00 & 0.98 \\
$\mathrm{~T}_{20}\left({ }^{\prime}\right.$ Bhagyashree') & 5.02 & 4.95 & 4.87 & 4.99 & 1.03 & 1.00 \\
$\mathrm{~T}_{21}\left({ }^{\prime}\right.$ Dhanashree') & 5.10 & 4.81 & 5.21 & 5.00 & 0.98 & 0.96 \\
$\mathrm{~T}_{22}(87-2 \times 18-1-1)$ & 3.90 & 4.32 & 3.48 & 3.85 & 1.12 & 1.12 \\
$\mathrm{~T}_{23}(87-2 \times 18-1-1)$ & 4.24 & 4.00 & 4.37 & 4.21 & 0.97 & 0.95 \\
$\mathrm{~T}_{24}(87-2 \times 18-1-1)$ & 4.55 & 4.95 & 3.87 & 4.11 & 1.18 & 1.21 \\
$\mathrm{~T}_{25}(87-2 \times 18-1-1)$ & 4.42 & 4.57 & 4.51 & 4.77 & 0.98 & 0.96 \\
$\mathrm{~T}_{26}(87-2 \times 18-1-1)$ & 5.23 & 4.99 & 5.39 & 5.09 & 0.97 & 0.98 \\
$\mathrm{~T}_{27}(87-2 \times 18-1-1)$ & 4.21 & 5.01 & 4.12 & 4.61 & 1.02 & 1.09 \\
$\mathrm{~T}_{28}(87-2 \times 18-1-1)$ & 5.22 & 5.79 & 5.54 & 5.84 & 0.94 & 1.00 \\
$\mathrm{~T}_{29}(87-2 \times 18-1-1)$ & 4.96 & 5.16 & 5.69 & 5.64 & 0.87 & 0.91 \\
$\mathrm{~T}_{30}(87-2 \times 18-1-1)$ & 4.39 & 4.77 & 4.50 & 4.77 & 0.97 & 1.00 \\
$\mathrm{~T}_{31}(87-2 \times 18-1-1)$ & 4.45 & 4.42 & 4.94 & 4.55 & 0.90 & 0.97 \\
$\mathrm{~T}_{32}(87-2 \times 18-1-1)$ & 4.49 & 4.36 & 4.88 & 4.47 & 0.92 & 0.98 \\
\hline $\mathrm{General} \mathrm{mean}$ & 4.55 & 4.81 & 4.49 & 4.74 & 1.02 & 1.02 \\
$\mathrm{~S} . \mathrm{E} \pm(m e a n)$ & 0.21 & 0.17 & 0.21 & 0.21 & 0.05 & 0.03 \\
$\mathrm{C} . \mathrm{D}$ at 5\% & 0.59 & 0.48 & 0.61 & 0.59 & 0.14 & 0.09 \\
\hline
\end{tabular}

\subsection{NUMBER OF LOCULES PER FRUIT}

The number of locules per fruit significantly differed in both generations (Table 2). The minimum number of locules per fruit was 2.4 ( $\mathrm{T}_{27}$ and $\mathrm{T}_{31}$, of cross 87-2 x 18-1-1) which was at par with $\mathrm{T}_{13}$ and $\mathrm{T}_{20}(2.47 \mathrm{~mm})$ and the maximum number of locules per fruit observed in $\mathrm{F}_{5}$ generation progenies in both crosses (M-3-1 x H-24 and 87-2 x 18-1-1) was 3.67 in $\mathrm{T}_{16}$ and $\mathrm{T}_{28}$ at par with $\mathrm{T}_{1}$ (3.4). In $\mathrm{F}_{6}$ generation, minimum number of locules was $2.0\left(\mathrm{~T}_{9}\right.$ and $\left.\mathrm{T}_{12}\right)$ at par with $\mathrm{T}_{31}$ (2.13) and the maximum locules was $3.73\left(\mathrm{~T}_{16}\right)$ at par with $\mathrm{T}_{28}$ (3.53). The mean numbers of locules per fruit were 2.89 and 2.63 in $\mathrm{F}_{5}$ and $\mathrm{F}_{6}$ generations, respectively. These results are in conformity with the work of Shibli et al. (1995), Sestras et al. (2006), Chakraborty et al. (2007), Hossain et al. (2010) in which they recorded the maximum polar and equatorial diameter in genotype TM-13(5.14 cm) and genotype BARI tomato $7(4.02 \mathrm{~cm})$, respectively, while the minimum polar and equatorial diameter was recorded in genotype TM-110 $(3.35 \mathrm{~cm})$ and TM-105(2.95 cm) respectively. Raina et al. (1980) and Randhawa et al. (1988) observed fruit shape index which ranged from 0.8 (cv.S1-120, which was flat round) to 1.7 (cv. Punjab Chhuhara, which was oval) in shape. Wagh (2002), Benal et al. (2005) and Mane et al. (2010) observed similar pericarp thickness. Kaur et al. (1976), Adsule et al. (1980), Garande (2006) and Mane et al. (2010) observed that round shaped varieties recorded more number of locules (3 to 6) than pear shaped cultivars which had 2 to 3 locules. 
Table 2: Physical fruit characters of tomato fruit

\begin{tabular}{|c|c|c|c|c|}
\hline \multirow[t]{2}{*}{ Treatment(T) } & \multicolumn{2}{|c|}{$\begin{array}{l}\text { Pericarp } \\
(\mathrm{mm})\end{array}$} & \multicolumn{2}{|c|}{$\begin{array}{l}\text { No. of } \\
\text { locules per fruit }\end{array}$} \\
\hline & $\mathrm{F}_{5}$ & $\mathrm{~F}_{6}$ & $\mathrm{~F}_{5}$ & $\mathrm{~F}_{6}$ \\
\hline $\mathrm{T}_{1}(\mathrm{M}-3-1 \times \mathrm{H}-24)$ & 5.20 & 5.50 & 3.40 & 2.67 \\
\hline $\mathrm{T}_{2}(\mathrm{M}-3-1 \times \mathrm{H}-24)$ & 5.47 & 4.50 & 2.80 & 3.40 \\
\hline $\mathrm{T}_{3}(\mathrm{M}-3-1 \times \mathrm{H}-24)$ & 5.00 & 4.90 & 3.20 & 2.20 \\
\hline $\mathrm{T}_{4}(\mathrm{M}-3-1 \times \mathrm{H}-24)$ & 4.67 & 5.10 & 3.20 & 2.20 \\
\hline $\mathrm{T}_{5}(\mathrm{M}-3-1 \times \mathrm{H}-24)$ & 4.93 & 4.63 & 2.87 & 2.40 \\
\hline $\mathrm{T}_{6}(\mathrm{M}-3-1 \times \mathrm{H}-24)$ & 5.87 & 6.03 & 3.00 & 2.53 \\
\hline $\mathrm{T}_{7}(\mathrm{M}-3-1 \times \mathrm{H}-24)$ & 5.40 & 4.70 & 2.87 & 3.07 \\
\hline $\mathrm{T}_{8}(\mathrm{M}-3-1 \times \mathrm{H}-24)$ & 5.80 & 5.30 & 3.00 & 2.20 \\
\hline $\mathrm{T}_{9}(\mathrm{M}-3-1 \times \mathrm{H}-24)$ & 4.73 & 4.20 & 2.67 & 2.00 \\
\hline $\mathrm{T}_{10}(\mathrm{M}-3-1 \times \mathrm{H}-24)$ & 4.80 & 4.17 & 2.80 & 3.00 \\
\hline $\mathrm{T}_{11}(\mathrm{M}-3-1 \times \mathrm{H}-24)$ & 4.87 & 4.17 & 2.87 & 2.60 \\
\hline $\mathrm{T}_{12}(\mathrm{M}-3-1 \times \mathrm{H}-24)$ & 4.20 & 4.60 & 2.67 & 2.00 \\
\hline $\mathrm{T}_{13}(\mathrm{M}-3-1 \times \mathrm{H}-24)$ & 5.47 & 4.70 & 2.47 & 2.33 \\
\hline $\mathrm{T}_{14}(\mathrm{M}-3-1 \times \mathrm{H}-24)$ & 4.47 & 4.77 & 2.60 & 2.27 \\
\hline $\mathrm{T}_{15}(\mathrm{M}-3-1 \times \mathrm{H}-24)$ & 4.47 & 4.03 & 3.33 & 3.20 \\
\hline $\mathrm{T}_{16}(\mathrm{M}-3-1 \times \mathrm{H}-24)$ & 5.13 & 5.23 & 3.67 & 3.73 \\
\hline $\mathrm{T}_{17}(\mathrm{M}-3-1 \times \mathrm{H}-24)$ & 4.93 & 4.97 & 3.07 & 3.40 \\
\hline $\mathrm{T}_{18}(\mathrm{M}-3-1 \times \mathrm{H}-24)$ & 4.47 & 4.43 & 3.27 & 3.13 \\
\hline $\mathrm{T}_{19}(\mathrm{M}-3-1 \times \mathrm{H}-24)$ & 4.00 & 4.90 & 2.53 & 2.93 \\
\hline $\mathrm{T}_{20}$ ('Bhagyashree') & 5.20 & 5.70 & 2.47 & 2.47 \\
\hline $\mathrm{T}_{21}$ ('Dhanashree') & 5.60 & 5.27 & 2.67 & 2.53 \\
\hline $\mathrm{T}_{22}(87-2 \times 18-1-1)$ & 3.80 & 4.43 & 2.60 & 2.27 \\
\hline $\mathrm{T}_{23}(87-2 \times 18-1-1)$ & 4.87 & 4.03 & 2.60 & 2.33 \\
\hline $\mathrm{T}_{24}(87-2 \times 18-1-1)$ & 4.40 & 4.13 & 2.67 & 2.53 \\
\hline $\mathrm{T}_{25}(87-2 \times 18-1-1)$ & 4.70 & 5.50 & 2.80 & 2.33 \\
\hline $\mathrm{T}_{26}(87-2 \times 18-1-1)$ & 5.07 & 5.17 & 2.53 & 2.47 \\
\hline $\mathrm{T}_{27}(87-2 \times 18-1-1)$ & 3.80 & 4.73 & 2.40 & 2.20 \\
\hline $\mathrm{T}_{28}(87-2 \times 18-1-1)$ & 4.53 & 5.37 & 3.67 & 3.53 \\
\hline $\mathrm{T}_{29}(87-2 \times 18-1-1)$ & 4.93 & 5.70 & 3.33 & 3.33 \\
\hline $\mathrm{T}_{30}(87-2 \times 18-1-1)$ & 4.60 & 5.57 & 3.13 & 2.33 \\
\hline $\mathrm{T}_{31}(87-2 \times 18-1-1)$ & 5.27 & 5.63 & 2.40 & 2.13 \\
\hline $\mathrm{T}_{32}(87-2 \times 18-1-1)$ & 5.00 & 5.30 & 2.80 & 2.33 \\
\hline General mean & 4.86 & 4.92 & 2.89 & 2.63 \\
\hline S.E $\pm($ mean $)$ & 0.31 & 0.20 & 0.23 & 0.20 \\
\hline C.D. at $5 \%$ & 0.89 & 0.55 & 0.64 & 0.58 \\
\hline
\end{tabular}

\subsection{FRUIT JUICE}

Juice of tomato fruits was significantly differed in $\mathrm{F}_{5}$ generation (Table 3 ). The maximum juice was 83.2 per cent $\left(\mathrm{T}_{25}\right.$, cross $\left.87-2 \times 18-1-1\right)$ at par with $\mathrm{T}_{19}$ (82.87 per cent) while the minimum was in $\mathrm{T}_{22}\left(63.2\right.$ per cent) at par with $\mathrm{T}_{27}\left(68.37\right.$ per cent). In $\mathrm{F}_{6}$ generation there were no significant differences among progenies. The juice per cent ranged from 78.28 ( $\mathrm{T}_{16}$, cross $\mathrm{M}-3-1$ 
$\mathrm{x} \mathrm{H}-24)$ to $89.51\left(\mathrm{~T}_{25}\right.$, cross $87-2$ x 18-1-1). The mean juice per cent was 77.37 and 82.72 in $\mathrm{F}_{5}$ and $\mathrm{F}_{6}$ generations, respectively. The standard checks 'Bhagyashree' $\left(\mathrm{T}_{20}\right)$ and 'Dhanashree' $\left(\mathrm{T}_{21}\right)$ had juice per cent of 81.77 and 81.8 in $\mathrm{F}_{5}$ and 83.56 and 80.31 in $\mathrm{F}_{6}$ generations, respectively. The variations in juice per cent could be attributed due to the differences in genotypes and fruit shapes. This finding is in accordance with the work of Roy and Choudhary (1972), Kalra and Nath (1992) and Garande (2006) who reported similar ranges in juice content of different tomato cultivars.

\subsection{TOTAL SOLUBLE SOLIDS}

As shown in Table 3, the total soluble solids (TSS) of tomato fruits significantly differed in both generations. The content of TSS ranged from 3.93 $\left(\mathrm{T}_{7}\right)$ to $5.03^{\circ}$ Brix $\left(\mathrm{T}_{18}\right)$ in $\mathrm{F}_{5}$ generation, while it varied from $4.31\left(\mathrm{~T}_{14}\right)$ to $5.17^{\circ} \mathrm{Brix}\left(\mathrm{T}_{27}\right.$ and $\left.\mathrm{T}_{29}\right)$ in $\mathrm{F}_{6}$ generation. The mean value of TSS were $4.69\left(\mathrm{~F}_{5}\right.$ generation) and $4.8^{\circ} \mathrm{Brix}\left(\mathrm{F}_{6}\right.$ generation). The differences in total soluble solids were due to variations in genotypes and environmental conditions prevailed during the growing seasons. Similar ranges of total soluble solids content were recorded by Randhawa et al. (1988), Wagh (2002) and Garande (2006).

Table 3: Biochemical composition of tomato fruit

\begin{tabular}{|c|c|c|c|c|c|c|}
\hline \multirow[t]{2}{*}{ Treatment(T) } & \multicolumn{2}{|c|}{$\begin{array}{l}\text { Fruit juice } \\
(\%)\end{array}$} & \multicolumn{2}{|c|}{$\begin{array}{l}\text { TSS } \\
\left({ }^{\circ} \text { Brix }\right)\end{array}$} & \multicolumn{2}{|c|}{$\begin{array}{l}\text { Titratable } \\
\text { Acidity }(\%)\end{array}$} \\
\hline & $\mathrm{F}_{5}$ & $\mathrm{~F}_{6}$ & $\mathrm{~F}_{5}$ & $\mathrm{~F}_{6}$ & $\mathrm{~F}_{5}$ & $\mathrm{~F}_{6}$ \\
\hline $\mathrm{T}_{1}(\mathrm{M}-3-1 \times \mathrm{H}-24)$ & 81.63 & 81.57 & 4.57 & 4.74 & 0.44 & 0.45 \\
\hline $\mathrm{T}_{2}(\mathrm{M}-3-1 \times \mathrm{H}-24)$ & 81.13 & 82.05 & 4.73 & 4.94 & 0.51 & 0.60 \\
\hline $\mathrm{T}_{3}(\mathrm{M}-3-1 \times \mathrm{H}-24)$ & 72.93 & 81.94 & 4.83 & 4.87 & 0.45 & 0.59 \\
\hline $\mathrm{T}_{4}(\mathrm{M}-3-1 \times \mathrm{H}-24)$ & 82.73 & 82.36 & 4.73 & 4.81 & 0.57 & 0.61 \\
\hline $\mathrm{T}_{5}(\mathrm{M}-3-1 \times \mathrm{H}-24)$ & 73.43 & 83.89 & 4.77 & 4.74 & 0.55 & 0.66 \\
\hline $\mathrm{T}_{6}(\mathrm{M}-3-1 \times \mathrm{H}-24)$ & 79.47 & 82.29 & 4.80 & 4.81 & 0.52 & 0.50 \\
\hline $\mathrm{T}_{7}(\mathrm{M}-3-1 \times \mathrm{H}-24)$ & 78.87 & 83.17 & 3.93 & 4.67 & 0.58 & 0.48 \\
\hline $\mathrm{T}_{8}(\mathrm{M}-3-1 \times \mathrm{H}-24)$ & 81.37 & 86.05 & 4.87 & 5.14 & 0.51 & 0.47 \\
\hline $\mathrm{T}_{9}(\mathrm{M}-3-1 \times \mathrm{H}-24)$ & 69.87 & 79.22 & 4.50 & 4.81 & 0.56 & 0.56 \\
\hline $\mathrm{T}_{10}(\mathrm{M}-3-1 \times \mathrm{H}-24)$ & 79.73 & 80.59 & 4.17 & 4.67 & 0.59 & 0.60 \\
\hline $\mathrm{T}_{11}(\mathrm{M}-3-1 \times \mathrm{H}-24)$ & 70.83 & 82.81 & 4.53 & 4.47 & 0.49 & 0.59 \\
\hline $\mathrm{T}_{12}(\mathrm{M}-3-1 \times \mathrm{H}-24)$ & 73.93 & 82.46 & 4.70 & 4.67 & 0.58 & 0.67 \\
\hline $\mathrm{T}_{13}(\mathrm{M}-3-1 \times \mathrm{H}-24)$ & 74.03 & 80.27 & 4.70 & 4.74 & 0.60 & 0.51 \\
\hline $\mathrm{T}_{14}(\mathrm{M}-3-1 \times \mathrm{H}-24)$ & 82.33 & 82.51 & 5.00 & 4.31 & 0.57 & 0.59 \\
\hline $\mathrm{T}_{15}(\mathrm{M}-3-1 \times \mathrm{H}-24)$ & 75.73 & 82.07 & 4.63 & 4.61 & 0.51 & 0.56 \\
\hline $\mathrm{T}_{16}(\mathrm{M}-3-1 \times \mathrm{H}-24)$ & 77.00 & 78.28 & 4.70 & 4.74 & 0.58 & 0.57 \\
\hline $\mathrm{T}_{17}(\mathrm{M}-3-1 \times \mathrm{H}-24)$ & 79.53 & 81.12 & 4.63 & 4.57 & 0.51 & 0.57 \\
\hline $\mathrm{T}_{18}(\mathrm{M}-3-1 \times \mathrm{H}-24)$ & 74.90 & 81.01 & 5.03 & 4.54 & 0.59 & 0.58 \\
\hline $\mathrm{T}_{19}(\mathrm{M}-3-1 \times \mathrm{H}-24)$ & 82.87 & 79.51 & 4.60 & 4.61 & 0.52 & 0.49 \\
\hline $\mathrm{T}_{20}$ ('Bhagyashree') & 81.77 & 83.56 & 4.70 & 5.04 & 0.58 & 0.54 \\
\hline $\mathrm{T}_{21}$ ('Dhanashree') & 81.80 & 80.31 & 4.80 & 4.81 & 0.46 & 0.49 \\
\hline $\mathrm{T}_{22}(87-2 \times 18-1-1)$ & 63.20 & 81.10 & 4.20 & 4.54 & 0.40 & 0.55 \\
\hline $\mathrm{T}_{23}(87-2 \times 18-1-1)$ & 80.67 & 83.78 & 4.87 & 4.87 & 0.52 & 0.58 \\
\hline $\mathrm{T}_{24}(87-2 \times 18-1-1)$ & 80.73 & 83.89 & 4.80 & 4.84 & 0.52 & 0.68 \\
\hline
\end{tabular}




\begin{tabular}{lllllll}
$\mathrm{T}_{25}(87-2 \times 18-1-1)$ & 83.20 & 89.51 & 4.80 & 5.07 & 0.50 & 0.58 \\
$\mathrm{~T}_{26}(87-2 \times 18-1-1)$ & 80.90 & 85.17 & 4.93 & 5.11 & 0.52 & 0.54 \\
$\mathrm{~T}_{27}(87-2 \times 18-1-1)$ & 68.37 & 80.50 & 4.90 & 5.17 & 0.53 & 0.59 \\
$\mathrm{~T}_{28}(87-2 \times 18-1-1)$ & 80.53 & 85.00 & 4.73 & 4.74 & 0.53 & 0.55 \\
$\mathrm{~T}_{29}(87-2 \times 18-1-1)$ & 76.43 & 81.28 & 4.90 & 5.17 & 0.48 & 0.48 \\
$\mathrm{~T}_{30}(87-2 \times 18-1-1)$ & 75.23 & 87.54 & 4.80 & 5.07 & 0.52 & 0.60 \\
$\mathrm{~T}_{31}(87-2 \times 18-1-1)$ & 71.33 & 85.67 & 4.50 & 4.64 & 0.55 & 0.44 \\
$\mathrm{~T}_{32}(87-2 \times 18-1-1)$ & 79.27 & 86.64 & 4.67 & 5.14 & 0.53 & 0.52 \\
\hline General mean & 77.37 & 82.72 & 4.69 & 4.80 & 0.53 & 0.56 \\
S.E \pm (mean $)$ & 2.68 & 3.04 & 0.14 & 0.10 & 0.03 & 0.04 \\
C.D. at 5\% & 7.58 & $\mathrm{NS}$ & 0.39 & 0.28 & 0.08 & 0.11 \\
\hline
\end{tabular}

\subsection{TITRATABLE ACIDITY}

The data presented in Table 3 revealed that there were significant differences among genotypes in titratable acidity both in $\mathrm{F}_{5}$ and $\mathrm{F}_{6}$ generations. The titratable acidity ranged from $0.4\left(\mathrm{~T}_{22}\right.$, cross 87-2 x 18-1-1) to 0.6 per cent ( $\mathrm{T}_{13}$, cross $\left.\mathrm{M}-3-1 \times \mathrm{H}-24\right)$ in $\mathrm{F}_{5}$ and $0.44\left(\mathrm{~T}_{31}\right.$, cross $87-2 \mathrm{x}$ 18-1-1) to 0.68 per cent $\left(\mathrm{T}_{24}\right.$, cross $\left.87-2 \times 18-1-1\right)$ in $\mathrm{F}_{6}$ generation. The mean value of titratable acidity were $0.53\left(\mathrm{~F}_{5}\right)$ and 0.56 per cent $\left(\mathrm{F}_{6}\right)$. Organic acids, especially citric and malic acids are the major organic acids found in tomato, contributing to more than 10 per cent of the dry solid content of tomatoes. Citric acid was reported to contribute to approximately 40-90 per cent of the total acidity in ripe tomatoes depending on the varieties. The results obtained in this study are in line with the work of Ringane (1992), Sestras et al. (2006), Turhan and Seniz (2009) who reported similar per cent titratable acidity in different tomato cultivars.

\section{8.pH}

The $\mathrm{pH}$ of fresh tomato juice as indicated in Table 4 reveals that the data were statistically significant among the genotypes in both generations. In $\mathrm{F}_{5}$ generation, significantly lowest $\mathrm{pH}$ (4.07) was recorded in standard checks 'Dhanashree' $\left(\mathrm{T}_{21}\right)$ which was at par with $\mathrm{T}_{6}(4.09)$ and significantly highest $\mathrm{pH}$ was 4.4 ( $\mathrm{T}_{23}$, cross $\left.87-2 \times 18-1-1\right)$ which was at par with $\mathrm{T}_{3}$ (4.36). While in $\mathrm{F}_{6}$ generation, the lowest $\mathrm{pH}$ was 4.07 in $\mathrm{T}_{6}$ which was at par with $\mathrm{T}_{8}$ and $\mathrm{T}_{25}(4.08)$ and the highest $\mathrm{pH}$ was 4.39 in $\mathrm{T}_{2}$ which was at par with $\mathrm{T}_{24}$ (4.34). The mean $\mathrm{pH}$ values were 4.22 and 4.20 in $\mathrm{F}_{5}$ and $\mathrm{F}_{6}$ generations, respectively. The difference in $\mathrm{pH}$ possibly attributed to the genotypes differences and environmental conditions prevailed during tomato production. Similar findings were reported by Garande (2006), Mane et al. (2010) who recorded pH range from 3.92 to 4.51 in winter grown tomato genotypes.

\subsection{ASCORBIC ACID}

The ascorbic acid content of tomato fruit significantly varied among the genotypes in both generations. The data presented in Table 4 depicted that the ascorbic acid content varied from $21.53 \mathrm{mg} / 100 \mathrm{~g}\left(\mathrm{~T}_{28}\right)$ to $36.27 \mathrm{mg} / 100 \mathrm{~g}\left(\mathrm{~T}_{26}\right)$ both in Cross 87-2 x 18-1-1 of $\mathrm{F}_{5}$ generation. While the ascorbic acid value observed in $F_{6}$ generation ranged from $22.31 \mathrm{mg} / 100 \mathrm{~g}$ 'Dhanashree' to $32.93 \mathrm{mg} / 100 \mathrm{~g}$ ( $\mathrm{T}_{32}$, cross of 87-2 x 18-1-1). The mean value was found 29.53 and $27.93 \mathrm{mg} / 100 \mathrm{~g}$ in $\mathrm{F}_{5}$ and $\mathrm{F}_{6}$ generations, respectively. High ascorbic acid improves 
nutrition, aids in better retention of natural colour and flavour of tomato products. The high content of ascorbic acid in these genotypes, which might be due to genesis of genotypes derived from L. peruvianum (18-1-1), which is richest source of ascorbic acid, which might have possibly contributed to the presence of high ascorbic acids in addition to favourable growing conditions. The present study results are in agreement with the findings reported by Sestras et al. (2006), Chakraborty et al. (2007), Mane et al. (2010), Dar and Sharma (2011) who recorded the ascorbic acid content in the range of 15.83 to $37.8 \mathrm{mg} / 100 \mathrm{~g}$.

Table 4: Biochemical composition of tomato fruit

\begin{tabular}{|c|c|c|c|c|}
\hline \multirow[b]{2}{*}{ Treatment(T) } & \multicolumn{2}{|c|}{ Fruit juice $\mathrm{pH}$} & \multicolumn{2}{|c|}{ Ascorbic acid $(\mathrm{mg} / 100 \mathrm{~g})$} \\
\hline & $\mathrm{F}_{5}$ & $\mathrm{~F}_{6}$ & $\mathrm{~F}_{5}$ & $\mathrm{~F}_{6}$ \\
\hline $\mathrm{T}_{1}(\mathrm{M}-3-1 \times \mathrm{H}-24)$ & 4.30 & 4.30 & 24.93 & 26.55 \\
\hline $\mathrm{T}_{2}(\mathrm{M}-3-1 \times \mathrm{H}-24)$ & 4.21 & 4.39 & 28.33 & 24.96 \\
\hline $\mathrm{T}_{3}(\mathrm{M}-3-1 \times \mathrm{H}-24)$ & 4.36 & 4.13 & 27.20 & 28.83 \\
\hline $\mathrm{T}_{4}(\mathrm{M}-3-1 \times \mathrm{H}-24)$ & 4.30 & 4.18 & 22.04 & 27.53 \\
\hline $\mathrm{T}_{5}(\mathrm{M}-3-1 \times \mathrm{H}-24)$ & 4.24 & 4.32 & 23.23 & 28.33 \\
\hline $\mathrm{T}_{6}(\mathrm{M}-3-1 \times \mathrm{H}-24)$ & 4.09 & 4.07 & 32.30 & 27.85 \\
\hline $\mathrm{T}_{7}(\mathrm{M}-3-1 \times \mathrm{H}-24)$ & 4.20 & 4.27 & 32.30 & 30.89 \\
\hline $\mathrm{T}_{8}(\mathrm{M}-3-1 \times \mathrm{H}-24)$ & 4.17 & 4.08 & 34.57 & 26.35 \\
\hline $\mathrm{T}_{9}(\mathrm{M}-3-1 \times \mathrm{H}-24)$ & 4.26 & 4.34 & 30.03 & 29.60 \\
\hline $\mathrm{T}_{10}(\mathrm{M}-3-1 \times \mathrm{H}-24)$ & 4.26 & 4.20 & 30.03 & 27.73 \\
\hline $\mathrm{T}_{11}(\mathrm{M}-3-1 \times \mathrm{H}-24)$ & 4.26 & 4.23 & 28.33 & 26.00 \\
\hline $\mathrm{T}_{12}(\mathrm{M}-3-1 \times \mathrm{H}-24)$ & 4.25 & 4.24 & 24.70 & 30.00 \\
\hline $\mathrm{T}_{13}(\mathrm{M}-3-1 \times \mathrm{H}-24)$ & 4.24 & 4.18 & 28.90 & 30.35 \\
\hline $\mathrm{T}_{14}(\mathrm{M}-3-1 \times \mathrm{H}-24)$ & 4.24 & 4.21 & 34.00 & 27.76 \\
\hline $\mathrm{T}_{15}(\mathrm{M}-3-1 \times \mathrm{H}-24)$ & 4.26 & 4.24 & 28.90 & 30.41 \\
\hline $\mathrm{T}_{16}(\mathrm{M}-3-1 \times \mathrm{H}-24)$ & 4.15 & 4.24 & 34.00 & 26.33 \\
\hline $\mathrm{T}_{17}(\mathrm{M}-3-1 \times \mathrm{H}-24)$ & 4.15 & 4.24 & 27.20 & 31.09 \\
\hline $\mathrm{T}_{18}(\mathrm{M}-3-1 \times \mathrm{H}-24)$ & 4.19 & 4.21 & 32.30 & 24.27 \\
\hline $\mathrm{T}_{19}(\mathrm{M}-3-1 \times \mathrm{H}-24)$ & 4.14 & 4.22 & 32.87 & 26.35 \\
\hline $\mathrm{T}_{20}$ ('Bhagyashree') & 4.13 & 4.15 & 30.60 & 23.31 \\
\hline $\mathrm{T}_{21}$ ('Dhanashree') & 4.07 & 4.10 & 29.47 & 22.31 \\
\hline $\mathrm{T}_{22}(87-2 \times 18-1-1)$ & 4.11 & 4.19 & 30.60 & 28.19 \\
\hline $\mathrm{T}_{23}(87-2 \times 18-1-1)$ & 4.40 & 4.22 & 24.93 & 27.92 \\
\hline $\mathrm{T}_{24}(87-2 \times 18-1-1)$ & 4.34 & 4.34 & 30.03 & 27.16 \\
\hline $\mathrm{T}_{25}(87-2 \times 18-1-1)$ & 4.21 & 4.08 & 33.43 & 30.59 \\
\hline $\mathrm{T}_{26}(87-2 \times 18-1-1)$ & 4.22 & 4.13 & 36.27 & 30.29 \\
\hline $\mathrm{T}_{27}(87-2 \times 18-1-1)$ & 4.35 & 4.22 & 20.40 & 26.24 \\
\hline $\mathrm{T}_{28}(87-2 \times 18-1-1)$ & 4.23 & 4.16 & 21.53 & 26.07 \\
\hline $\mathrm{T}_{29}(87-2 \times 18-1-1)$ & 4.26 & 4.22 & 30.03 & 24.96 \\
\hline $\mathrm{T}_{30}(87-2 \times 18-1-1)$ & 4.14 & 4.13 & 32.87 & 32.29 \\
\hline $\mathrm{T}_{31}(87-2 \times 18-1-1)$ & 4.19 & 4.16 & 34.30 & 30.36 \\
\hline $\mathrm{T}_{32}(87-2 \times 18-1-1)$ & 4.13 & 4.09 & 34.30 & 32.93 \\
\hline General mean & 4.22 & 4.2 & 29.53 & 27.93 \\
\hline S.E $\pm($ mean $)$ & 0.04 & 0.04 & 1.23 & 1.58 \\
\hline C.D. at $5 \%$ & 0.10 & 0.11 & 3.48 & 4.46 \\
\hline
\end{tabular}




\subsection{FRUIT SUGARS}

Data on tomatoes fruit sugars content is presented in Table 5. As it is depicted in the table there were significant differences among progenies in respect to reducing, non-reducing and total sugars content of tomato fruits in both the years.

\subsubsection{REDUCING SUGARS}

Reducing sugars significantly varied from $2.19\left(\mathrm{~T}_{22}\right)$ to 2.97 per cent $\left(\mathrm{T}_{2}\right)$ in $\mathrm{F}_{5}$ generation and from $2.08\left(\mathrm{~T}_{28}\right)$ to 2.79 per cent $\left(\mathrm{T}_{6}\right)$ in $\mathrm{F}_{6}$ generation. The mean reducing sugars were observed $2.62\left(\mathrm{~F}_{5}\right)$ and 2.39 per cent $\left(\mathrm{F}_{6}\right)$. The two standard checks, 'Bhagyashree' and 'Dhanashree' had 2.71 and 2.62 per cent in $F_{5}$ and 2.49 and 2.37 per cent in $F_{6}$ generations, respectively (Table 5).

\subsubsection{NON-REDUCING SUGAR}

There were significant differences in non-reducing sugar content among progenies in both the trials (Table 5). In the first trial significantly more non-reducing sugar (0.95 per cent) was recorded in $\mathrm{T}_{32}$ (cross of $\left.87-2 \times 18-1-1\right)$ which was at par with $\mathrm{T}_{25}$ (0.89 per cent) and significantly lowest non-reducing sugar was in $\mathrm{T}_{17}(0.38$ per cent $)$ which was at par with $\mathrm{T}_{11}$ ( 0.40 per cent).While in the second trial, $\mathrm{T}_{21}$ ('Dhanashree') recorded significantly highest nonreducing sugar(1.01 per cent) and significantly lowest non-reducing sugar was observed in $\mathrm{T}_{29}$ (0.69 per cent) which was at par with $\mathrm{T}_{31}(0.75$ per cent $)$.

\subsubsection{TOTAL SUGARS}

The content of total sugar of tomatoes fruit significantly varied from 2.94 (T27, cross of 87-2 x 18-1-1) to 3.72 per cent (T8, cross of M-3-1 x H-24) in F5 generation (Table 5) while in F6 generation it varied from 2.89 (T28, cross 87-2 x 18-1-1) to 3.71 per cent (T6, cross of M-3-1 x $\mathrm{H}-24$ ). The mean value of total sugars were 3.3 (F5) and 3.25 per cent (F6) generations. The standard checks had 3.45 and 3.36 per cent 'Bhagyashree' and 3.48 and 3.38 per cent 'Dhanashree' total sugars in F5 and F6 generations, in their order.

Sugars and organic acids content have been reported to be most responsible for the pleasant sweet and sour taste of tomatoes. Sugars, primarily glucose and fructose, contribute to about 50 per cent of the dry matter content in tomatoes. Of the sugars identified in tomato, fructose probably was the most important compound that produced the characteristic sweetness. Sugar content substantially increased during the ripening period of the fruits. The work carried out by Sestras et al. (2006) indicated that higher the content of total sugars in fruits higher was titratable acid and ascorbic acid.

The variations in sugars content of tomato genotypes might perhaps brought by differences in genotypes and its interaction with growth resources. These findings are in conformity with the work done by Adsule et al. (1980), Sandhu et al. (1990) and Ringane (1992) who reported the total sugars in the range of 2.18 to 4.44 . Both reducing and non-reducing sugars are found in tomato, about 50 to 65 per cent of tomato solids consisted of reducing sugars. The content of reducing sugars observed was ranged from 2.14 to 2.97 per cent while non-reducing sugar ranged from 0.4 to 1.01 per cent. These observations are in agreement with findings reported by Garande (2006) and Hossain et al. (2010). 
Table 5: Biochemical composition of tomato fruit

\begin{tabular}{|c|c|c|c|c|c|c|}
\hline \multirow[t]{2}{*}{ Treatment(T) } & \multirow{2}{*}{$\begin{array}{l}\text { Reducing } \\
(\%)\end{array}$} & \multirow{2}{*}{$\begin{array}{l}\text { sugars } \\
\mathrm{F}_{6}\end{array}$} & \multicolumn{2}{|c|}{$\begin{array}{l}\text { Non-reducing } \\
\text { sugar }(\%)\end{array}$} & \multicolumn{2}{|c|}{$\begin{array}{l}\text { Total sugars } \\
(\%)\end{array}$} \\
\hline & & & $\mathrm{F}_{5}$ & $\mathrm{~F}_{6}$ & $\mathrm{~F}_{5}$ & $\mathrm{~F}_{6}$ \\
\hline $\mathrm{T}_{1}(\mathrm{M}-3-1 \times \mathrm{H}-24)$ & 2.83 & 2.17 & 0.70 & 0.90 & 3.53 & 3.08 \\
\hline $\mathrm{T}_{2}(\mathrm{M}-3-1 \times \mathrm{H}-24)$ & 2.97 & 2.52 & 0.42 & 0.90 & 3.39 & 3.42 \\
\hline $\mathrm{T}_{3}(\mathrm{M}-3-1 \times \mathrm{H}-24)$ & 2.73 & 2.49 & 0.70 & 0.93 & 3.43 & 3.42 \\
\hline $\mathrm{T}_{4}(\mathrm{M}-3-1 \times \mathrm{H}-24)$ & 2.71 & 2.40 & 0.65 & 0.93 & 3.36 & 3.34 \\
\hline $\mathrm{T}_{5}(\mathrm{M}-3-1 \times \mathrm{H}-24)$ & 2.60 & 2.33 & 0.65 & 0.90 & 3.25 & 3.23 \\
\hline $\mathrm{T}_{6}(\mathrm{M}-3-1 \times \mathrm{H}-24)$ & 2.80 & 2.79 & 0.75 & 0.92 & 3.55 & 3.71 \\
\hline $\mathrm{T}_{7}(\mathrm{M}-3-1 \times \mathrm{H}-24)$ & 2.52 & 2.35 & 0.49 & 0.88 & 3.01 & 3.23 \\
\hline $\mathrm{T}_{8}(\mathrm{M}-3-1 \times \mathrm{H}-24)$ & 2.91 & 2.76 & 0.81 & 0.88 & 3.72 & 3.64 \\
\hline $\mathrm{T}_{9}(\mathrm{M}-3-1 \times \mathrm{H}-24)$ & 2.86 & 2.48 & 0.56 & 0.91 & 3.42 & 3.39 \\
\hline $\mathrm{T}_{10}(\mathrm{M}-3-1 \times \mathrm{H}-24)$ & 2.52 & 2.36 & 0.52 & 0.86 & 3.04 & 3.22 \\
\hline $\mathrm{T}_{11}(\mathrm{M}-3-1 \times \mathrm{H}-24)$ & 2.78 & 2.14 & 0.40 & 0.82 & 3.18 & 2.96 \\
\hline $\mathrm{T}_{12}(\mathrm{M}-3-1 \times \mathrm{H}-24)$ & 2.39 & 2.37 & 0.69 & 0.84 & 3.08 & 3.21 \\
\hline $\mathrm{T}_{13}(\mathrm{M}-3-1 \times \mathrm{H}-24)$ & 2.66 & 2.20 & 0.61 & 0.87 & 3.27 & 3.08 \\
\hline $\mathrm{T}_{14}(\mathrm{M}-3-1 \times \mathrm{H}-24)$ & 2.40 & 2.34 & 0.62 & 0.89 & 3.01 & 3.22 \\
\hline $\mathrm{T}_{15}(\mathrm{M}-3-1 \times \mathrm{H}-24)$ & 2.33 & 2.36 & 0.67 & 0.85 & 3.00 & 3.21 \\
\hline $\mathrm{T}_{16}(\mathrm{M}-3-1 \times \mathrm{H}-24)$ & 2.52 & 2.35 & 0.84 & 0.85 & 3.36 & 3.20 \\
\hline $\mathrm{T}_{17}(\mathrm{M}-3-1 \times \mathrm{H}-24)$ & 2.77 & 2.23 & 0.38 & 0.92 & 3.15 & 3.15 \\
\hline $\mathrm{T}_{18}(\mathrm{M}-3-1 \times \mathrm{H}-24)$ & 2.84 & 2.44 & 0.52 & 0.89 & 3.37 & 3.33 \\
\hline $\mathrm{T}_{19}(\mathrm{M}-3-1 \times \mathrm{H}-24)$ & 2.45 & 2.32 & 0.74 & 0.82 & 3.19 & 3.14 \\
\hline $\mathrm{T}_{20}$ ('Bhagyashree') & 2.71 & 2.49 & 0.74 & 0.87 & 3.45 & 3.36 \\
\hline $\mathrm{T}_{21}$ ('Dhanashree') & 2.62 & 2.37 & 0.86 & 1.01 & 3.48 & 3.38 \\
\hline $\mathrm{T}_{22}(87-2 \times 18-1-1)$ & 2.19 & 2.40 & 0.88 & 0.83 & 3.07 & 3.24 \\
\hline $\mathrm{T}_{23}(87-2 \times 18-1-1)$ & 2.45 & 2.26 & 0.85 & 0.93 & 3.30 & 3.19 \\
\hline $\mathrm{T}_{24}(87-2 \times 18-1-1)$ & 2.29 & 2.20 & 0.77 & 0.79 & 3.06 & 2.99 \\
\hline $\mathrm{T}_{25}(87-2 \times 18-1-1)$ & 2.59 & 2.60 & 0.89 & 0.87 & 3.48 & 3.47 \\
\hline $\mathrm{T}_{26}(87-2 \times 18-1-1)$ & 2.83 & 2.69 & 0.75 & 0.82 & 3.55 & 3.51 \\
\hline $\mathrm{T}_{27}(87-2 \times 18-1-1)$ & 2.32 & 2.14 & 0.62 & 0.82 & 2.94 & 2.96 \\
\hline $\mathrm{T}_{28}(87-2 \times 18-1-1)$ & 2.53 & 2.08 & 0.51 & 0.81 & 3.04 & 2.89 \\
\hline $\mathrm{T}_{29}(87-2 \times 18-1-1)$ & 2.95 & 2.41 & 0.49 & 0.69 & 3.44 & 3.10 \\
\hline $\mathrm{T}_{30}(87-2 \times 18-1-1)$ & 2.70 & 2.40 & 0.85 & 0.83 & 3.55 & 3.23 \\
\hline $\mathrm{T}_{31}(87-2 \times 18-1-1)$ & 2.51 & 2.32 & 0.76 & 0.75 & 3.27 & 3.07 \\
\hline $\mathrm{T}_{32}(87-2 \times 18-1-1)$ & 2.65 & 2.58 & 0.95 & 0.89 & 3.55 & 3.47 \\
\hline General mean & 2.62 & 2.39 & 0.68 & 0.87 & 3.30 & 3.25 \\
\hline S.E $\pm($ mean $)$ & 0.10 & 0.08 & 0.08 & 0.04 & 0.11 & 0.10 \\
\hline C.D. at $5 \%$ & 0.29 & 0.23 & 0.23 & 0.13 & 0.32 & 0.29 \\
\hline
\end{tabular}

\subsection{LYCOPENE}

Tomatoes constitute the major dietary source of lycopene. The data with regard to lycopene content is presented in Table 6 which revealed that there were significant differences among the genotypes in this investigation. In $\mathrm{F}_{5}$ generation, significantly maximum lycopene content (5.95 $\mathrm{mg} / 100 \mathrm{~g}$ ) was recorded in $\mathrm{T}_{26}$ of cross $87-2 \times 18-1-1$ and significantly minimum lycopene (2.0 
$\mathrm{mg} / 100 \mathrm{~g}$ ) was in $\mathrm{T}_{11}$ of cross M-3-1 x H-24. Likewise, significantly maximum lycopene content $(4.23 \mathrm{mg} / 100 \mathrm{~g})$ was registered in $\mathrm{T}_{26}$ and the minimum $(2.42 \mathrm{mg} / 100 \mathrm{~g})$ in $\mathrm{T}_{28}$ in $\mathrm{F}_{6}$ progeny generation. The two standard checks 'Bhagyashree' and 'Dhanashree' had lycopene 3.54 and $3.26 \mathrm{mg} / 100 \mathrm{~g}$ in $\mathrm{F}_{5}$ and 3.35 and $3.17 \mathrm{mg} / 100 \mathrm{~g}$ in $\mathrm{F}_{6}$, respectively. Lycopene is the most abundant carotene in red tomato fruit accounting for up to 90 per cent of the total carotenoids (Radzevicius et al., 2009). The lycopene content was significantly varied from 2.0 to 5.95 $\mathrm{mg} / 100 \mathrm{~g}$ in $\mathrm{F}_{5}$ and 2.42 to $4.23 \mathrm{mg} / 100 \mathrm{~g}$ in $\mathrm{F}_{6}$ generations (Table 6). Similar ranges in lycopene content were registered by Ringane (1992), Garande (2006), Dar and Sharma (2011). The fruit colour of the tomato genotypes used in this investigation had mainly orange red and less dark red colour with intermediate lycopene content. The results of this finding are in close agreement with the work of Wagh (2002) and Garande (2006) who confirmed that the orange coloured tomato cultivars contain low lycopene than red fruited cultivars. Moreover, Wagh (2002) and Garande (2006) found that lower lycopene content was observed in rabi season grown tomatoes.

\subsection{2. $\beta$-CAROTENE}

The result of $\beta$-carotene content was significantly differed among the genotypes tested in both the years (Table 6). In $\mathrm{F}_{5}$, significantly highest value of $\beta$-carotene was observed in $\mathrm{T}_{32}(2.97$ $\mathrm{mg} / 100 \mathrm{~g})$ which was at par with $\mathrm{T}_{26}(2.85 \mathrm{mg} / 100 \mathrm{~g})$ in cross of 87-2 x 18-1-1 and significantly lowest $\beta$-carotene was in $\mathrm{T}_{7}(1.02 \mathrm{mg} / 100 \mathrm{~g})$ in cross of M-3-1 $\mathrm{x} \mathrm{H}-24$ which was at par with $\mathrm{T}_{4}$ $(1.04 \mathrm{mg} / 100 \mathrm{~g})$. Similarly, in $\mathrm{F}_{6}$, the maximum $\beta$-carotene was noted in $\mathrm{T}_{32}(2.94 \mathrm{mg} / 100 \mathrm{~g})$ which was at par with $\mathrm{T}_{25}(2.8 \mathrm{mg} / 100 \mathrm{~g})$ and the minimum $\beta$-carotene recorded in $\mathrm{T}_{7}(1.11$ $\mathrm{mg} / 100 \mathrm{~g})$ which was at par with $\mathrm{T}_{28}(1.2 \mathrm{mg} / 100 \mathrm{~g})$. The mean value of $\beta$-carotene was 1.94 and $1.98 \mathrm{mg} / 100 \mathrm{~g}$ in $\mathrm{F}_{5}$ and $\mathrm{F}_{6}$ generations, respectively. The significant differences observed in $\beta$-carotene content may be brought by differences in the genotypes (orange and dark red colour fruit). The results of this study are in accordance with the work of Wagh (2002), Garande (2006), Radzevicius et al. (2009) and Dar and Sharma (2011) who reported similar range of $\beta$-carotene content in different tomato genotypes/cultivars.

Table 6: Biochemical composition of tomato fruit

\begin{tabular}{lllll}
\hline & \multicolumn{2}{l}{$\begin{array}{l}\text { Lycopene } \\
\text { Treatment }(\mathrm{T})\end{array}$} & \multicolumn{3}{l}{$\begin{array}{l}\text { 3-carotene } \\
(\mathrm{mg} / 100 \mathrm{~g})\end{array}$} \\
\cline { 2 - 5 } & $\mathrm{F}_{5}$ & $\mathrm{~F}_{6}$ & $\mathrm{~F}_{5}$ & $\mathrm{~F}_{6}$ \\
\hline $\mathrm{T}_{1}(\mathrm{M}-3-1 \times \mathrm{H}-24)$ & 2.12 & 3.22 & 1.70 & 1.76 \\
$\mathrm{~T}_{2}(\mathrm{M}-3-1 \times \mathrm{H}-24)$ & 3.41 & 2.92 & 1.80 & 1.86 \\
$\mathrm{~T}_{3}(\mathrm{M}-3-1 \times \mathrm{H}-24)$ & 3.20 & 3.18 & 2.14 & 2.16 \\
$\mathrm{~T}_{4}(\mathrm{M}-3-1 \times \mathrm{H}-24)$ & 2.62 & 3.02 & 1.04 & 1.40 \\
$\mathrm{~T}_{5}(\mathrm{M}-3-1 \times \mathrm{H}-24)$ & 2.44 & 3.13 & 2.25 & 2.08 \\
$\mathrm{~T}_{6}(\mathrm{M}-3-1 \times \mathrm{H}-24)$ & 3.73 & 3.48 & 2.46 & 2.63 \\
$\mathrm{~T}_{7}(\mathrm{M}-3-1 \times \mathrm{H}-24)$ & 2.64 & 2.89 & 1.02 & 1.11 \\
$\mathrm{~T}_{8}(\mathrm{M}-3-1 \times \mathrm{H}-24)$ & 3.82 & 3.39 & 2.62 & 2.19 \\
$\mathrm{~T}_{9}(\mathrm{M}-3-1 \times \mathrm{H}-24)$ & 2.55 & 2.88 & 1.51 & 1.50 \\
$\mathrm{~T}_{10}(\mathrm{M}-3-1 \times \mathrm{H}-24)$ & 2.54 & 2.78 & 1.71 & 1.79 \\
$\mathrm{~T}_{11}(\mathrm{M}-3-1 \times \mathrm{H}-24)$ & 2.00 & 2.53 & 1.21 & 1.26 \\
$\mathrm{~T}_{12}$ (M-3-1 x H-24) & 2.40 & 2.74 & 1.36 & 1.46 \\
$\mathrm{~T}_{13}$ (M-3-1 x H-24) & 2.35 & 2.65 & 1.45 & 1.48
\end{tabular}




\begin{tabular}{|c|c|c|c|c|}
\hline $\mathrm{T}_{14}(\mathrm{M}-3-1 \times \mathrm{H}-24)$ & 2.29 & 2.54 & 1.31 & 1.40 \\
\hline $\mathrm{T}_{15}(\mathrm{M}-3-1 \times \mathrm{H}-24)$ & 3.38 & 3.16 & 1.74 & 1.81 \\
\hline $\mathrm{T}_{16}(\mathrm{M}-3-1 \times \mathrm{H}-24)$ & 3.61 & 3.03 & 1.75 & 1.90 \\
\hline $\mathrm{T}_{17}(\mathrm{M}-3-1 \times \mathrm{H}-24)$ & 2.57 & 2.92 & 1.68 & 1.75 \\
\hline $\mathrm{T}_{18}(\mathrm{M}-3-1 \times \mathrm{H}-24)$ & 2.82 & 3.04 & 1.91 & 2.01 \\
\hline $\mathrm{T}_{19}(\mathrm{M}-3-1 \times \mathrm{H}-24)$ & 2.61 & 2.92 & 1.44 & 1.67 \\
\hline $\mathrm{T}_{20}$ ('Bhagyashree') & 3.54 & 3.35 & 2.08 & 1.97 \\
\hline $\mathrm{T}_{21}$ ('Dhanashree') & 3.26 & 3.17 & 2.37 & 2.12 \\
\hline $\mathrm{T}_{22}(87-2 \times 18-1-1)$ & 3.65 & 3.18 & 2.22 & 2.20 \\
\hline $\mathrm{T}_{23}(87-2 \times 18-1-1)$ & 2.70 & 3.01 & 2.42 & 2.38 \\
\hline $\mathrm{T}_{24}(87-2 \times 18-1-1)$ & 2.44 & 2.86 & 2.59 & 2.64 \\
\hline $\mathrm{T}_{25}(87-2 \times 18-1-1)$ & 3.45 & 3.35 & 2.82 & 2.80 \\
\hline $\mathrm{T}_{26}(87-2 \times 18-1-1)$ & 5.95 & 4.23 & 2.85 & 2.79 \\
\hline $\mathrm{T}_{27}(87-2 \times 18-1-1)$ & 2.93 & 3.12 & 1.90 & 2.14 \\
\hline $\mathrm{T}_{28}(87-2 \times 18-1-1)$ & 2.20 & 2.42 & 1.06 & 1.20 \\
\hline $\mathrm{T}_{29}(87-2 \times 18-1-1)$ & 2.65 & 2.92 & 1.97 & 2.03 \\
\hline $\mathrm{T}_{30}(87-2 \times 18-1-1)$ & 2.87 & 2.79 & 2.40 & 2.44 \\
\hline $\mathrm{T}_{31}(87-2 \times 18-1-1)$ & 2.34 & 2.85 & 2.37 & 2.32 \\
\hline $\mathrm{T}_{32}(87-2 \times 18-1-1)$ & 3.57 & 3.28 & 2.97 & 2.94 \\
\hline General mean & 2.95 & 3.03 & 1.94 & 1.98 \\
\hline S.E $\pm($ mean $)$ & 0.21 & 0.17 & 0.20 & 0.16 \\
\hline C.D. at $5 \%$ & 0.59 & 0.49 & 0.56 & 0.45 \\
\hline
\end{tabular}

\section{CONCLUSIONS AND RECOMMENDATIONS}

Study was conducted to evaluate $\mathrm{F}_{5}$ and $\mathrm{F}_{6}$ generations of tomato hybrid derivatives for its physicochemical characters and its suitability for processing. The $\mathrm{F}_{5}$ and $\mathrm{F}_{6}$ generations of two crosses (M-3-1 x H-24 and 87-2 x 18-1-1) and the two standard checks ('Bhagyashree' and 'Dhanashree') were evaluated for processing suitability.

The results showed significant differences among the treatments. The tested tomato progenies had minimum physical and biochemical properties to be used in processing. Significantly highest polar and equatorial diameters, shape index of fruit, pericarp thickness were recorded in both $\mathrm{F}_{5}$ and $\mathrm{F}_{6}$ generations. Biochemical composition and processing qualities of tomato genotypes in both $F_{5}$ and $F_{6}$ generations showed significant differences except fruit juice per cent in $F_{6}$ generation. Major processing quality characters maximum recorded in $\mathrm{F}_{5}$ and $\mathrm{F}_{6}$ generations, total soluble solids, 5.03 and $5.17^{\circ}$ Brix; titratable acidity 0.6 per cent and 0.68 per cent; low pH, 4.07 and 4.07; maximum ascorbic acid, 36.27 and $32.93 \mathrm{mg} / 100 \mathrm{~g}$; fruit total sugars, 3.72 per cent and 3.71 per cent; maximum lycopene 5.95 and $4.23 \mathrm{mg} / 100 \mathrm{~g}$; $\beta$-carotene, 2.97 and 2.94 $\mathrm{mg} / 100 \mathrm{~g}$ in $\mathrm{F}_{5}$ and $\mathrm{F}_{6}$ progenies generations, respectively.

It can be concluded that the crosses of M-3-1 x H-24 and 87-2 x 18-1-1 progenies showed superior characters than the two standard checks and could be used for processing purposes. Thus, the progenies indicated as $\mathrm{T}_{6}, \mathrm{~T}_{26}$ and $\mathrm{T}_{32}$ may be used as best progenies in the processing industry as they fulfilled the minimum requirements of total soluble solids, $\mathrm{pH}$, ascorbic acid, lycopene and $\beta$-carotene content. 


\section{ACKNOWLEDGEMETS}

The Authors would like to thank All India Coordinated Research Project on Vegetable Crops and Tomato Improvement Scheme, Mahatma Phule Agricultural University, Rahuri for materials and financial support.

\section{REFERENCES}

[1] Adsule P.G. Amba, D., Tikko S.K., and Pal, A.B. Studies on the Evaluation of Tomato Cultivars for Making Juice. Indian Food Packer, Vol 34 No.1, 1980, 18-20.

[2] A.O.A.C. Official Methods of Analysis of the Association of Official Analytical Chemists, 15thed. Washigton, D.C.1990.

[3] Balasubramanian, T. Studies On Quality and Nutritional Aspects of Tomato. J. Food Sci. Technol. 21, 1984, 419-421.

[4] Benal, B.P., Madalageri, M.B. and Rokhade, A.K. Physico-Chemical Qualities of Fruits of Commercial Tomato Hybrids and Varieties in Ghatprabha Left Bank Command Area Processing In To Puree. J. Food Sci. and Technol, Vol. 42 No. 1, 2005, 8-11.

[5] Chakraborty, I., Vanlalliani.,Chattopadhyay A. and Hazra P. Studies On Processing and Nutritional Qualities of Tomato as Influenced by Genotypes and Environment. Veg. Sci. Vol. 34 No. 1, 2007, 26-31.

[6] Dar, R.A. and Sharma, J.P. Genetic Variability Studies of Yield and Quality Traits in Tomato (Solanum lycopersicum L.). Int. J. Plant Breed. Genet. Vol. 5, 2011, 168-174. Http://Scialert.Net/Abstract/103923.Ijpbg.2011.168.174

[7] Garande, V.K. Evaluation of Orange Fruited Tomato Cultivars for Beta Carotene Content in Fresh Fruit and Processed Product. PhD. Thesis Submitted To Mahatma Phule Krishi Vidyapeeth, Rahuri.2006.

[8] Gomez, K.A. and Gomez, A.A. Statistical Procedures For Agricultural. 2nd Ed. John Wiley and Sons, Inc. UK. 1984.

[9] Hossain, M.E., Alam, M. J., Hakim, M.A., Amanullah, A.S.M., and Ahsanullah, A.S.M. An Assessment of Physicochemical Properties of Some Tomato Genotypes and Varieties Grown at Rangpur. Bangladesh Res. Pub. J. Vol.4, No. 3, 2010, 235-243.

[10] Http://Www.Bdresearchpublications.Com/Admin/Journal/Upload/09180/09180.Pdf

[11] Kalra, R. And Nath, N. Effect of Variety on Morphological and Physico-Chemical Characteristics of Tomatoes. Beverage and Food World, Vol.19, No. 4, 1992, 26-27.

[12] Kaur, G., Jaiswal, S.P., Kanwar, J.S. and Nandpuri, K.S. Variability in Certain PhysicoChemical Characters of Tomato. Indian Food Packer, Vol. 30, No. 6, 1976, 5-9.

[13] Mane, R., Sridevi, O.,Salimath, P.M., Deshpande, S.K., Khot, A.B. Performance Stability of Different Tomato Genotypes. Indian Journal of Agricultural Sciences, Vol .80 No. 10, 2010, 898-901.

[14] Radzevicius,A., Karkleliene, R., Bobinas, C., and Viskelis, P. Nutrition Quality of Different Tomato Cultivars. Zemdirbyste-Agric., Vol. 96, No. 3, 2009, 67-75.

[15] Http://Www.Lzi.It/Tomai/96(3)Tomas/96-3tomas-67-75.Pdf.

[16] Raina, B.L., Kalra, C.L.,Teotia, M.S., Rodrigues, R.,Nandpuri, K.S., Kanwar, J.S. and Singh, S.1980. Studies on Suitability of Some Tomato Varieties for Canning.Veg.Sci., Vol. 7, No. 1, 1980,60-66. 
[17] Randhawa, K.S., Saimbhi, M.S., and Gill, B.S. Commercial Evaluation of Tomato Varieties for Processing. Veg. Sci. Vol.15, No. 2, 1988, 181-184.

[18] Ranganna, S. Handbook of Analysis and Quality Control for Fruit and Vegetable Products. 2nd Eds., Tata Mcgraw-Hill Publishing Company Ltd., New Delhi.1986.

[19] Ringane, N.B. Evaluation of Tomato Cultivars for Processing Quality. M.Sc. Thesis. Mahatma Phule Agricultural University. 1992.

[20] Roy, S.K. And Choudhary, B. Studies On Physico-Chemical Characteristics of Few Varieties of Tomato in Relation to Processing. J. Food Sci. Tech.,Vol. 9, No. 3, 1972, 151-153.

[21] Sandhu, K.S., Cheema, D.S. and Singh, S. Studies on The Varietal Difference in PhysicoChemical Characteristics of Some Tomato Varieties. Beverage and Food World, Vol.17 No. 1, 1990, 34-35.

[22] Sestras A., Jidavu, M., Sestras, R., Apahidean, M., Harsan, E., Tamas E. and Gao, Y. The Response of Several Tomato Cultivars for Processing in Central Transylvania Conditions II. Fruit Quality. 2006.

[23] Http://Www.Notulaebotanicae.Ro/Nbha/Article/Viewfile/276/268.

[24] Sharma A.,Malhotra, S.R., Modgil, R. and Bhama, S. Tomato as a Nutrient Powerhouse and Most Widely Grown Vegetable in the World, Indian Food Pack.Vol. 2, No.9, 2008, 18-19.

[25] Shibli, R.A., Ereifej, K.I.,Ajlouni, M.A. and Hussain, A. Evaluation of Thirteen Open Pollinated Cultivars and Three Hybrids of Tomato. II. Physical Properties and Chemical Composition of Fruits. Pak. J. Agri. Sci., Vol.32, No. 2, 1995, 225-234.

[26] Http://Pakjas.Com.Pk/Upload/88872.Pdf

[27] Swamy, K.R.M. and Sadashiva, A.T. Tropical Vegetable Crops. In: Peter, K.V. And Abraham, Z. (Eds.) Biodiversity in Horticultural Crops.Vol. I. Daya Publishing House, India. 2007, 109-183.

[28] Tiwari, R.N.,Mishra, M., Choudhury, B. and Palai, S.K. Tomato. In: T. K. Bose,J. Kabir, T. K. Maity, V.A. Parthasarathy and M.G. Som (Eds.),Vegetable Crops .Vol. I. Third Ed. Naya Prokash Publishing, India. 2002, 1-154.

[29] Turhan, A. and Seniz, V. Estimation of Certain Chemical Constituents of Fruits Of Selected Tomato Genotypes Grown In Turkey. Afr. J. Agric. Res.Vol. 4, No. 10, 2009, 1086-1092. Http://Www.Ademicjournals.Org/Ajar/Pdf/202009/Oct/Turhnandsentz.Pdf.

[30] Wagh, R.S. Morpho-Physiological and Nutritional Assessment of Somatic Hybrid Derivatives of Tomato. PhD. Thesis, Submitted to Mahatma Phule Krishi Vidyapeeth, Rahuri (Maharashtra) India.2002. 